\title{
Simulation of Si-MOSFETs with the Mutation Operator Monte Carlo Method
}

\author{
JÜRGEN JAKUMEIT ${ }^{\mathrm{a}}$, AMANDA DUNCAN ${ }^{\mathrm{b}}$, UMBERTO RAVAIOLI $^{\mathrm{c}} *$ and KARL HESS ${ }^{\mathrm{d}}$

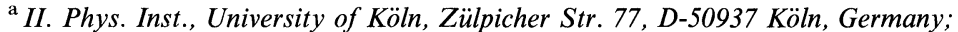 \\ ${ }^{\mathrm{b}}$ Intel Corporation, RA1-309, 5200 N.E. Elam Young Parkway, Hillsboro, OR 97124-6497; \\ ${ }^{\mathrm{c}}$ Beckman Institute, University of Illinois at Urbana-Champaign, 405 N. Mathews Avenue, Urbana, IL 61801, USA; \\ ${ }^{\mathrm{d}}$ Beckman Institute, University of Illinois at Urbana-Champaign, 405 N. Mathews Avenue, Urbana, IL 61801, USA
}

\begin{abstract}
The Mutation Operator Monte Carlo method (MOMC) is a new type of Monte Carlo technique for the study of hot electron related effects in semiconductor devices. The MOMC calculates energy distributions of electrons by a physical mutation of the distribution towards a stationary condition. In this work we compare results of an one dimensional simulation of an $800 \mathrm{~nm}$ Si-MOSFET with full band Monte Carlo calculations and measurement results. Starting from the potential distribution resulting from a drift diffusion simulation, the MOMC calculates electron distributions which are comparable to FBMC-results within minutes on a modern workstation. From these distributions, substrate and gate currents close to experimental results can be calculated. These results show that the MOMC is useful as a post-processor for the investigation of hot electron related problems in Si-MOSFETs. Beside the computational efficiency, a further advantage of the MOMC compared to standard MC techniques is the good resolution of the high energy tail of the distribution without the necessity of any statistical enhancement.
\end{abstract}

Keywords: Monte Carlo simulation, hot electrons

\section{INTRODUCTION}

During the last two years we have developed a new approach to hot electron related problems in $\mathrm{Si}$ MOSFETs, which is based on a mixture of Evolutionary optimization algorithms (EA) [1] and Monte Carlo (MC) technique [2]. The EA is used to optimize energy distributions of electrons towards an agreement with measurement results like substrate or gate currents. A major problem in this quasi-backward calculation of electron distributions is that many different distributions fit to one measured current value. It is very difficult or even impossible to force the EA towards physical correct distributions only by restrictions. We overcome this problem by using a Physical

\footnotetext{
*Corresponding author. Tel.: (217) 244-5765, Fax: (217) 244-4333. E-mail: ravaioli@uiuc.edu.
} 
Mutation Operator (PMO) within the EA. The PMO forces the EA to converge to distributions which are in agreement with the physical model of the PMO. The PMO itself is the basis for a novel type of Monte Carlo technique, the Mutation Operator Monte Carlo method (MOMC), which applies the PMO to an initial distribution repeatedly, until a stationary condition is reached. The effectiveness of the EA depends greatly on the quality of the PMO, which should be at the same time accurate and inexspensive. The present work focuses on specific details and applications of the MOMC itself. The approach is built on a relatively simple transport model which is calibrated with a traditional Monte Carlo simulation. We would like to stress that since the MOMC was conceived as the first approximation step for an EA approach, it cannot be as accurate in all details as the full band Monte Carlo. But when the parameters of the MOMC are well calibrated from Monte Carlo simulation results, as done here, the MOMC can then be used as stand-alone method when one needs a rapid approximate evaluation of hot carrier distribution. Ideally, this method should be used as a first order test, to select conditions for which a more costly Monte Carlo simulation should be run.

\section{MUTATION OPERATOR MONTE CARLO METHOD}

The main part of the MOMC is the PMO, which mutates an energy distribution of electrons by a Monte-Carlo-like simulation of the electron transport (see Fig. 1). The distribution in a small interval around a randomly choosen energy $E_{\text {start }}$ and position $x_{\text {start }}$ is associated to a MC-electron. The drift and scattering processes of this electron are simulated in the same way as in a standard MC-simulation, until a final energy $E_{\text {final }}$ and position $x_{\text {final }}$ are reached, and the electron density represented by the MC-electron is returned to the distribution. For the MC-simulation of the electron movement a simple one-valley effective mass

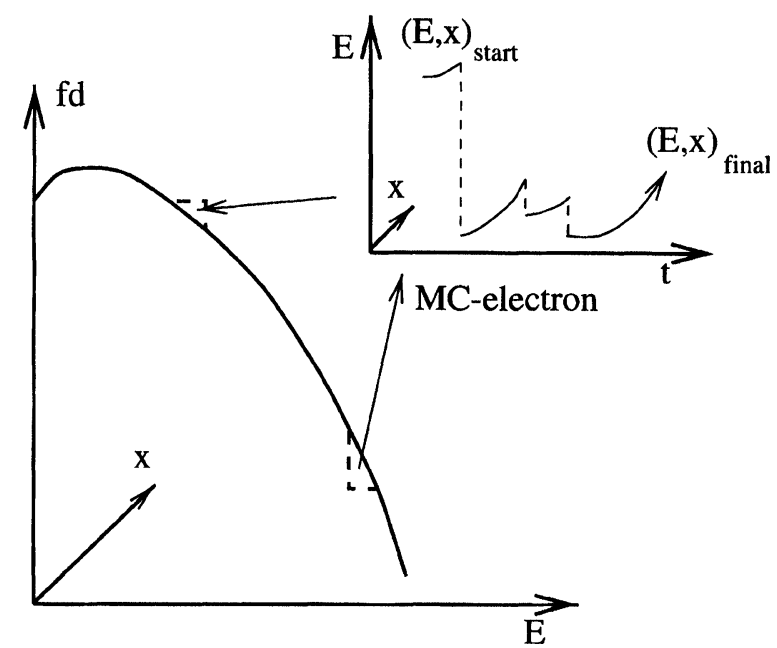

FIGURE 1 Principle of the physical mutation operator.

approach is used, taking the effective mass $m_{\text {eff }}$ as a free parameter. Scatterings due to phonon absorption, phonon emission and impact ionization are included in the model. The phonon scattering rates are calculated by a full band approach [3]. Instead of many rates for scattering between different valleys one averaged scattering rate for absorbtion and one for emission is used (see [2] for details). For impact ionization the rate computed in [4] was taken (assuming zero field and no collision broadening). The phonon scattering and the impact ionization rates are then multiplied by the factors $f_{\text {phonon }}$ and $f_{\text {impact }}$, respectively. Introduction of these two parameters gives us more flexibilty to calibrate the simple model by comparison with full band approaches.

The Monte Carlo simulation in the PMO needs the carrier density and electric field or potential profile in the simulation region as input (along the channel in case of Si-MOSFETs).

Since our main interest is to use the MOMC to calculate the high energy tail of the distribution, which has only a small impact on the total carrier distribution, in this approach the electric field and the carrier density are taken as inputs and Poisson's equation is not solved self-consistently.

From the electron distributions calculated by the MOMC substratecurrents $I_{\text {sub }}$ are calculated 
assuming that $I_{\text {sub }}$ is determined by the amount of holes created by impact ionization:

$$
I_{\text {sub }}=e \cdot \int_{\text {channel }} \int_{0}^{\infty} f d(E, x) W_{\mathrm{ii}}(E) d E d x
$$

where $e$ is the electron charge, $W_{\mathrm{ii}}(E)$ is the impact ionization rate and $f d(E, x)$ the energy distribution of electrons integrated over the depth of the simulated region (with electron distribution $f d(E$, $x$ ) we denote the distribution function times density of states).

The gate current $I_{\text {gate }}$ is estimated using a WKB approach with Schottky lowering of the barrier [5]:

$I_{\text {gate }}=K_{\text {hit }} \cdot e \cdot \int_{\text {channel }} \int_{0}^{\infty} f d(E, x) P_{\text {trans }}(E, x) d E d x$

where $P_{\text {trans }}(E, x)$ is the probability to cross the $\mathrm{SiO}_{2}$-layer. We assumed that the rate at which electrons trying to cross the $\mathrm{Si} / \mathrm{SiO}_{2}$ interface is proportional to the carrier density. The proportional constant $K_{\text {hit }}=7.5 \cdot 10^{-9} \mathrm{~s}^{-1}$ was calibrated by a comparison with FBMC and measurement results.

\section{RESULTS AND DISCUSSION}

In this work we present results from an one dimensional simulation of a $0.8 \mu \mathrm{m}$ Si-MOSFET, for which experimental data were published in the classic paper by $\mathrm{Ng}$ et al. [6]. The input for the MOMC, i.e. the carrier density and electric field profile, was calculated by a Full Band Monte Carlo (FBMC) program developed at the University of Illinois and by a HydroDynamic (HD) model (ISE AG, Zürich).

Figure 2 shows the electron distribution given by MOMC and FBMC for a drain voltage $V d=6 \mathrm{~V}$ and a gate voltage $V g=7 \mathrm{~V}$ in the region important for substrate and gate current generation, i.e. the crossover from channel to drain (the drain region starts at location $x=1 \mu \mathrm{m}$; source and substrate contacts are held at $0 \mathrm{~V}$ bias). MCMOMC denotes the MOMC results, which were

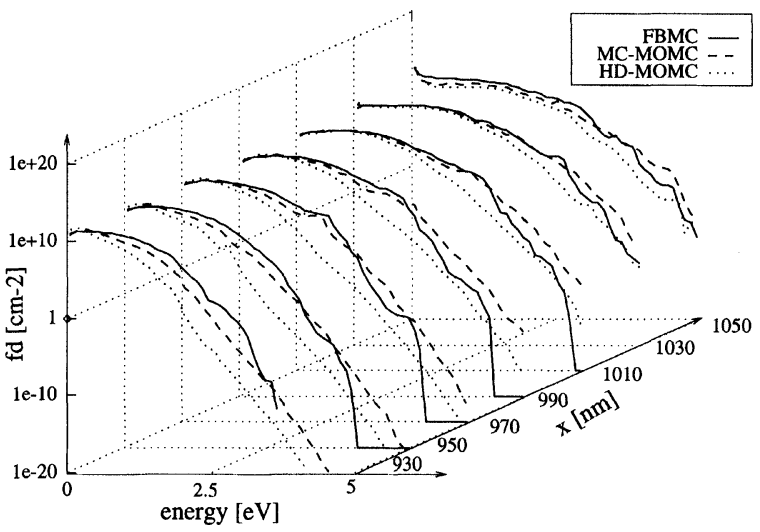

FIGURE 2 Energy distribution of electrons in a $800 \mathrm{~nm} \mathrm{Si-}$ $\operatorname{MOSFET}\left(V_{\text {drain }}=6 \mathrm{~V}, V_{\text {gate }}=7 \mathrm{~V}\right)$ at the crossover from channel to drain. MOMC results calculated from FBMC input (dashed line, MC-MOMC) and HD input (dotted line, HDMOMC) are compared with FBMC simulation results (solid line).

obtained using the FBMC results for carrier density and electric field as input, and HD-MOMC the MOMC results calculated on the basis of HDmodel input. MOMC results calculated from FBMC input are in fairly good agreement with the FBMC results, especially at the crossover between channel and drain at $1 \mu \mathrm{m}$. The HDMOMC distribution does not fit the FBMC results as well but is reasonably close for $x<1 \mu \mathrm{m}$.

The difference between HD-MOMC distributions and FBMC results for $x<1 \mu \mathrm{m}$ can be explained by looking at Figure 3. Here, the electric field and drift velocity are plotted as functions of $x$. The HD-model gives a smaller rise of the electric field for $x<0.9 \mu \mathrm{m}$ and then a steeper increase up to the peak at $1.01 \mu \mathrm{m}$, leading to a later heating of the electrons. This explains the lower electron distribution calculated from HD-MOMC input for $x<1 \mu \mathrm{m}$. The drift velocityprofiles from FBMC and MC-MOMC calculations are comparable. Both simulations yield a broad peak between $0.92 \mu \mathrm{m}$ and $1.02 \mu \mathrm{m}$. The HD-MOMC simulation leads to a sharper peak at $x=0.99 \mu \mathrm{m}$ reflecting the sharper peak of the electric field.

The good resolution of the high energy tail by the MOMC becomes obvious by looking at the 


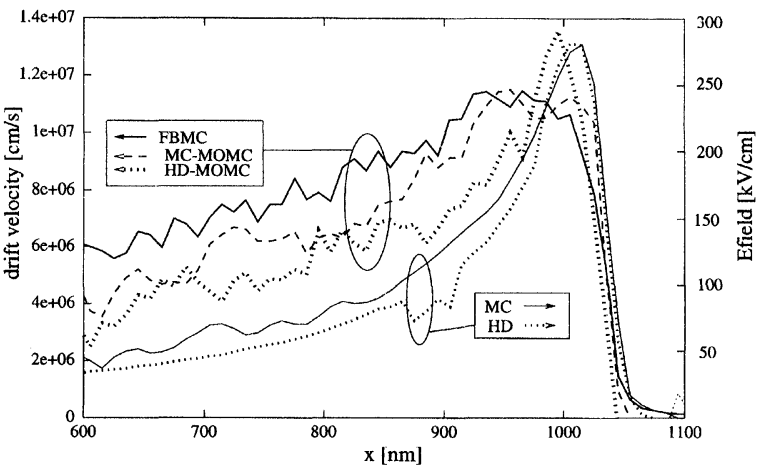

FIGURE 3 Drift velocity and electric field profile along the channel for an $800 \mathrm{~nm} \operatorname{Si}-\mathrm{MOSFET}\left(V_{\text {drain }}=6 \mathrm{~V}, V_{\text {gate }}=7 \mathrm{~V}\right)$ calculated by FBMC simulation (solid lines) and MOMC simulation using FBMC results (dashed lines) or HD-results (dotted lines) as input.

distributions above $4 \mathrm{eV}$ (Fig. 2). The FBMC result drops to zero when the carrier density goes below $1^{-10} \mathrm{~cm}^{-2}$. The MOMC resolves this regime with the same resultion as the low energy part. Theoretically, the resolution of the MOMC is independent of the order of magnitudes displayed. This could be proven by bulk simulations up to 80 orders of magnitude. A statistical enhancement, which was used in the FBMC simulation, is not necessary. Therefore, the MOMC could also be used as a post-processor for standard MC simulations, in order to avoid the computational complexity connected with statistical enhancement. Figure 4 compares substrate and gatecurrents calculated from the three different approaches with the measurement results of $\mathrm{Ng}$ et al. Obviously FBMC, MC-MOMC and HD-MOMC give an excellent agreement between measured and calculated substrate currents. For gate currents the agreement is not as good, showing differences up to one order of magnitude. Interestingly the gate currents calculated from FBMC distributions are not closer to the measurement results than those from MC-MOMC and HD-MOMC. It is not possible to judge from the comparison with measurement, which simulation approach yields the more realistic electron distributions.

The above results demonstrate that the MOMC gives energy distributions of electrons which are

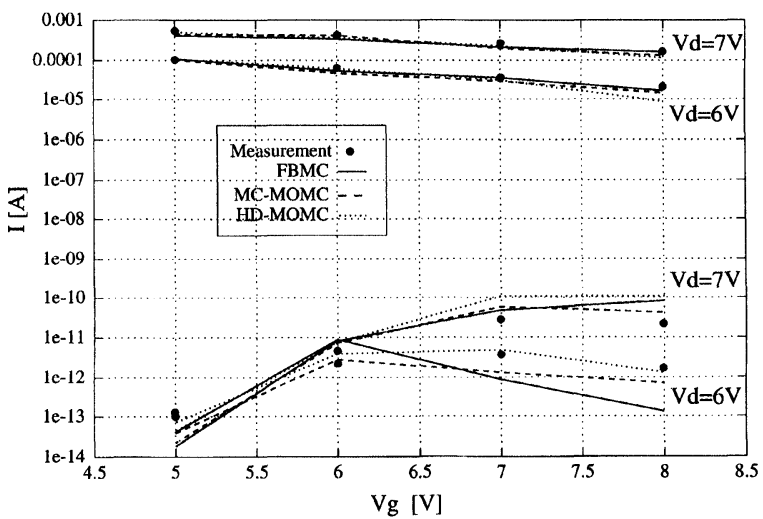

FIGURE 4 Comparison of substrate (top curves) and gate currents (bottom curves) calculated from FBMC, MC-MOMC and HD-MOMC results with experimental data from $\mathrm{Ng}$ et al. [5].

close to FBMC results and in agreement with measurement results even when HD-model calculations are used as input. For each $(V d, V g)$-pair in Figure 4, the MOMC calculates the corresponding distribution within several minutes on a HP735 workstation. Thus, the computational cost is comparable to HD-simulation and significantly less than FBMC calculations. This makes the MOMC a very interesting post processor for HDsimulations to gain insight on information like the energy distribution of carriers, without increasing the computational expense. The discussion above on the difference between FBMC and HD-MOMC distributions demonstrates also that such information is important for the interpretation of simulation results like the effect of slight changes in the electric field profile on the electron distribution and related hot electron effects.

\section{CONCLUSIONS}

A comparison of MOMC results with FBMC calculations and measurement results for a $800 \mu \mathrm{m}$ Si-MOSFET shows that the MOMC can be used as a fast post processor in device simulation. The method is based on a physical mutation operator which changes (mutates) electron distributions by a Monte Carlo like simulation of the electron 
movement using a simple effective mass model including electron-phonon scattering and impact ionization. Energy distributions of electrons calculated by the MOMC from hydrodynamic drift diffusion calculations were found to be comparable to FBMC results. The computational cost for the MOMC calculations is comparable to or smaller than the cost for hydrodynamic calculations. A next step is the application of the MOMC to short channel MOSFETs in the sub $100 \mathrm{~nm}$ range. Further improvements of the physical model, a nonparabolic approach to the bandstructure and additional scattering rates are planned.

\section{Acknowledgements}

This work was partially supported by the Deutsche Forschungsgemeinschaft contract No. JA853/1-1 (J.J.), the Semiconductor Research Corporation, Contract 96-CJ-816 (U.R and K.H.), and by fellowships from the US DOE Computational Science Graduate Fellowship Program and the Intel Foundation (A.D.).

\section{References}

[1] Jakumeit, J. (1995). "Genetic algorithms: A new approach to energy balance equations", Appl. Phys. Lett., 66(14), $1812-1814$.

[2] Jakumeit, J., Ravaioli, U. and Hess, K. (1996). "Calculation of hot electron distributions in silicon by means of an evolutionary algorithm", J. Appl. Phys., 80 (9), 5061 - 5066.

[3] Hess, K. (1991). Monte Carlo Device Simulation: Full Band and Beyond, Norwell, MA: Kluwer Academic Publishers.

[4] Bude, J., Hess, K. and Iafrate, G. J. (1992). "Impact ionization in semiconductors: Effects of high electric fields and high scattering rates", Phys. Rev. B., 45(19), 10958 10964.

[5] Huang, C., Wang, T., Chen, C. N., Chang, M. C. and Fu, J. (1992). "Modeling hot-electron gate current in $\mathrm{Si}$ MOSFET's using a coupled drift-diffusion and Monte Carlo method", IEEE Trans. on Electron Dev., 39(11), 2562-2568.
[6] Ng, K. K. and Taylor, G. W. (1983). "Effects of hot carrier trapping in $n$ - and $p$-channel MOSFET's", IEEE Trans. on Electron Dev., 30(8), 871-876.

\section{Authors' biographies}

Jürgen Jakumeit studied physics at the University of Cologne and finished his dissertation in 1994. His research concerned quantum size effects in semiconductor structures. Through two half year stays at the University of Michigan, Ann Arbor, MI, and the University of Illinois, UrbanaChampaign, IL, he got engaged in the field of device simulation. At the moment he works as Postdoc at the University of Cologne on the simulation of silicon MOSFETs and the investigation of quantum interference effects.

Amanda Duncan obtained the Ph.D. in Electrical Engineering at the University of Illinois, UrbanaChampaign, in 1996, with a dissertation on full band Monte Carlo simulation of Si devices. She is now a Senior Component Device Engineer with Intel Corporation, Hillsboro, Oregon.

Umberto Ravaioli is a Professor in the Department of Electrical and Computer Engineering at the University of Illinois at Urbana-Champaign. His main research interests are in the areas of semiconductor device physics and simulation, numerical methods, and high performance computing.

Karl Hess is a Swanlund Chair Professor in the Department of Electrical and Computer Engineering at the University of Illinois at UrbanaChampaign. His main research interests are in the areas of semiconductor device physics, quantum transport, and opto-electronics device simulation. 

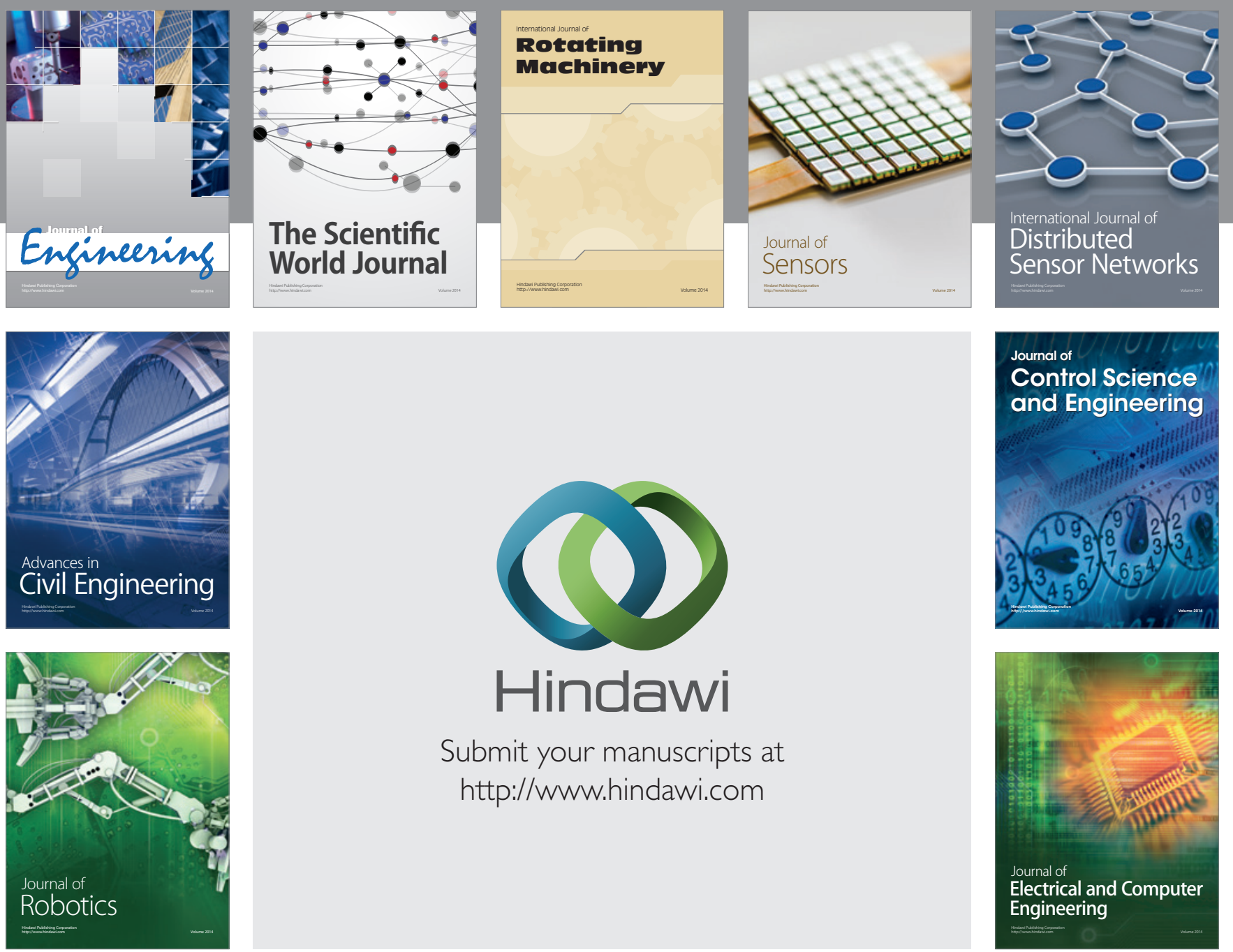

Submit your manuscripts at

http://www.hindawi.com
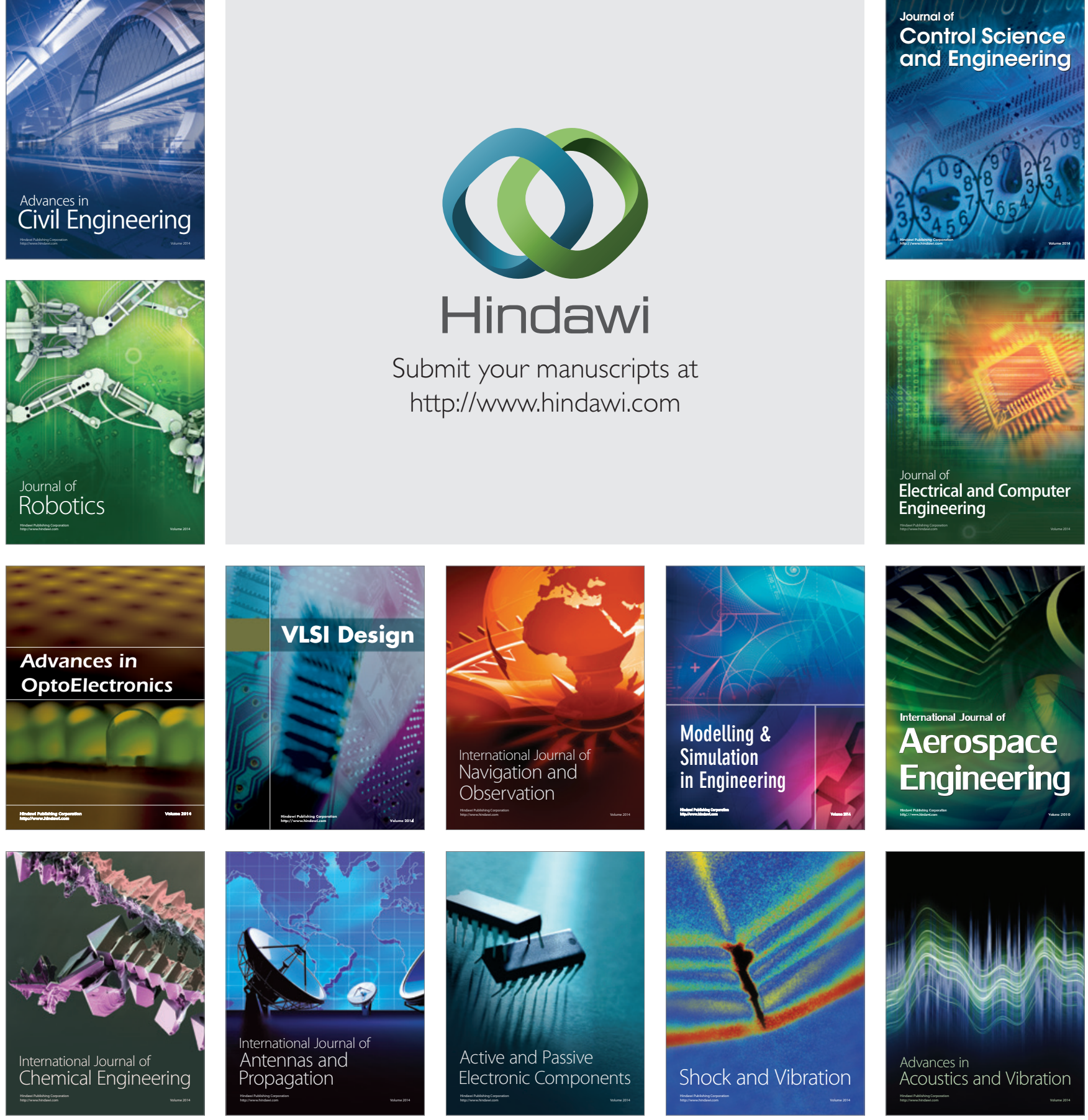